\title{
Investigación sobre el sistema de abastecimiento del antiguo alcázar de los arzobispos en Alcalá de Henares
}

\author{
Research on the supply system of the Alcazar of the archbishops in Alcalá de Henares
}

\author{
Enrique José Fernández Tapia a ${ }^{\text {, Irene de Bustamante Gutiérrez }}{ }^{\text {b }}$, Fernando Da Casa \\ Martín c \\ Universidad de Alcalá, Alcalá de Henares, Spain \\ a fernandez.tapia@uah.es; ${ }^{\mathrm{b}}$ irene.bustamante@uah.es; ${ }^{\mathrm{c}}$ fernando.dacasa@uah.es
}

\begin{abstract}
Seven years after the reconquest in 1118, Alkal'a Nahar (Alcalá de Henares) to Muslims, thanks to the Toledo archbishop, Bernardo de Sedirac, King Alfonso VII donates this population, to the archbishopric of Toledo. During the Muslim domination, a fortress with an important suburb had been built on the nearby hills, known as Alcalá la Vieja which, from that moment, was gradually abandoned, to settle on the plain, next to the old Church of San Justo. The archbishops were aware of the historical and religious importance of this site and with their policies, they were reinforcing and attracting more population. Most of the researchers think that it was Ximénez de Rada (1209-1247), who began the construction of the headquarters of the Toledo archbishops in Alcalá de Henares, formed by a fortress and a walled enclosure, probably over what there was it has been an extensive agricultural farm. Associated with this, there are news of the existence of waterwheel, from the Muslim era in this place. On the other hand, there is a reference that places around 1300, the construction of a viaje de agua for water supply. Little else was known about the supply system of the Alcazar. Thanks to the research carried out, we have learned that the construction of said infrastructure is based on the ancient Arab technique, known as qanat. These are mines that drain the aquifer, about two kilometers upstream, to the north and transport the water to the walled enclosure. The main advantages of this system of supply, is that the water is captured far from the fortification and can also be tubed and taking advantage of the existing slope, maintaining the pressure, to make the water sprout above ground level, forming sources.
\end{abstract}

Keywords: Alcalá de Henares, qanat, viaje de agua.

\section{Introducción histórica}

La fértil llanura escalonada de terrazas aluviales, situada en la ribera derecha y los cerros de la izquierda, forman, un típico valle asimétrico, que ha ofrecido a los pobladores históricos, de lo que hoy se llama Alcalá de Henares, posibilidades también asimétricas para la vida. Los cerros, que se alzan casi $300 \mathrm{~m}$ por encima del llano, ofrecen claras ventajas desde el punto de vista defensivo, frente a las terrazas de la llanura aluvial. La situación en el llano, por el contrario, se ve favorecida por la cercanía a las aguas del río, de las fuentes naturales y de las aguas de los acuíferos.

Esta dualidad, en las posibilidades para la vida, que ofrece el valle, ha sido uno de los principales debates estratégicos, en los que se han visto inmersos los antiguos pobladores del valle. 
La ciudad de Alcalá de Henares, llamada también Complutum, en época romana, ha ido moviéndose de ubicación a lo largo de su historia, alternando entre los cerros y el llano (entre una ribera y la otra), a lo largo de unos $5 \mathrm{~km}$, tomando como eje el río.

\subsection{El origen de la ciudad actual}

Los restos arqueológicos prehistóricos y romanos encontrados proporcionan muestras de una importante actividad agrícola en el llano. Por el contrario, en los cerros se sitúan los restos de antiguos castros prehistóricos, como el del cerro de San Juan del Viso, situado junto al río y que fue conquistado por los romanos hacia el siglo II a.C.. Como sucedió en otros muchos lugares, este emplazamiento fue aprovechado por los conquistadores para establecerse. La dificultad mayor de los cerros para alcanzar el agua, hizo que tuviesen que emplear diversas técnicas para abastecerse, muestra de ello son los restos, que permanecen, de un antiguo aljibe (Málaga, 1988).

Los romanos permanecieron en los cerros hasta el siglo I d.C., momento en el que se deciden a construir en la llanura, una ciudad, justo al otro lado del río, comunicadas ambas por un puente. La ciudad estuvo repartida entre ambas orillas durante muchos años. Pero a las ya mencionadas ventajas que ofrece el llano, hay que añadir que el nuevo asentamiento construido, recogía en su diseño el conocimiento técnico existente en la época y por el que los romanos son reconocidos. Complutum, municipio romano, era una ciudad amurallada, perfectamente planificada. Su traza forma una retícula ortogonal de cardos y decumanos, en la que encontramos el foro, con su basílica, el mercado, las termas, etc... El lugar elegido contaba con fuentes, conocidas hoy como de la Salud y del Juncal, también se han encontrado pozos y toda una planificada red de galerías y conducciones de agua para el saneamiento y abastecimiento de la ciudad (Sánchez, 2013). Complutum era el núcleo urbano principal del llano, por el que se repartían otros pequeños núcleos y villas agrícolas.

Las crónicas hablan de que a principios del siglo IV se produjo en Complutum, un importante acontecimiento religioso, que vino a cambiar la historia de la ciudad y a facilitarle una nueva ubicación. La historia a la que nos referimos es la de los Santos Niños Justo y Pastor, que fueron martirizados por no adjurar de su fe cristiana y enterrados, en uno de esos pequeños núcleos agrícolas, situado a unos $2 \mathrm{~km}$ al este. Con el tiempo se fue formando un nuevo núcleo, alrededor de una iglesia, construida en la zona del martirio, convirtiéndose en un importante foco de atracción religiosa, en los siglos posteriores (Vallejo, 1993). Este nuevo asentamiento cristiano fue amortizando a la ciudad romana, hasta que, en el siglo VIII, los musulmanes invadieron la península. En ese momento, los venerados restos de los mártires fueron trasladados, abandonando incluso la península Ibérica, pero el núcleo urbano permaneció como un pequeño burgo agrícola, donde seguramente siguió viviendo la población cristiana.

Los musulmanes plantearon una nueva estrategia y decidieron volver a aprovechar las posibilidades defensivas de los cerros, construyendo a principios del siglo $\mathrm{X}$ una fortaleza en ellos, aguas arriba de los anteriores asentamientos. Junto con el alcazar de Gudalajara, esta nueva fortaleza, servía para custodiar la parte baja del transitado valle del río Henares, paso natural desde el centro penínsular hacia Zaragoza y Cataluña. La fortaleza de Qal'at Abd Salam, que así se llamaba y que da nombre a la ciudad actual, ocupaba unas dos hectáreas y disponía de ocho cubos en su muralla. Contaba además con un importante arrabal, donde residía el grueso de la población musulmana. Ambos núcleos permanecieron unidos durante siglos por un puente, seguramente de madera (Pavón, 1982), hoy desaparecido. Al igual que el asentamiento romano del cerro de San Juan del Viso, Qal'at Abd Salam contaba también con aljibes.

El arzobispo de Toledo Bernardo de Sedirac, consciente de la importancia religiosa de Alcalá, llevó a cabo la conquista a los musulmanes, de la ciudad, tras un largo asedio, en 1118. Pocos años más tarde, en 1125, Alfonso VII cedió la ciudad a los arzobispos de Toledo, pasando de esta forma a ser estos los señores feudales de la misma (Vázquez, 1993). 


\section{Orígenes del alcázar de los arzobispos}

Según las teorías más aceptadas hoy día, los arzobispos de Toledo pudieron aprovechar, para establecer su residencia en la villa, una antigua almunia perteneciente a un rico hacendado musulmán, situada justo al norte de la zona del martirio y esta a su vez, podría ser heredera de una villa agrícola tardo-romana anterior. El conjunto agrícola que dispondría de casas, huerta, noria y acequias, se uniría a una hipotética torre vigía, avanzadilla de la fortaleza musulmana del cerro, formando así el germen de lo que más tarde evolucionaría para convertirse hacia el siglo XIII, en el alcázar de los arzobispos de Toledo (Consuegra, 2014), en el por entonces llamado Burgo de Santiuste, más tarde Alcalá de
Santiuste y finalmente Alcalá de Fenares o Henares.

La configuración inicial de la residencia se compondría: del alcázar, murallas y la albacarahuerta, que irán evolucionando con el paso del tiempo y las ampliaciones de diversos arzobispos, hasta convertirse en uno de los castillospalacio más impresionantes de nuestro país. Con un recinto amurallado, de más casi 6 ha, con 21 torres, de las que quedan 16 , está caracterizado en todo momento por su espléndida huerta, jardines y patios con sus fuentes (Fig. 1), abastecidos por una importante infraestructura hidráulica (Sánchez, et al., 2011).

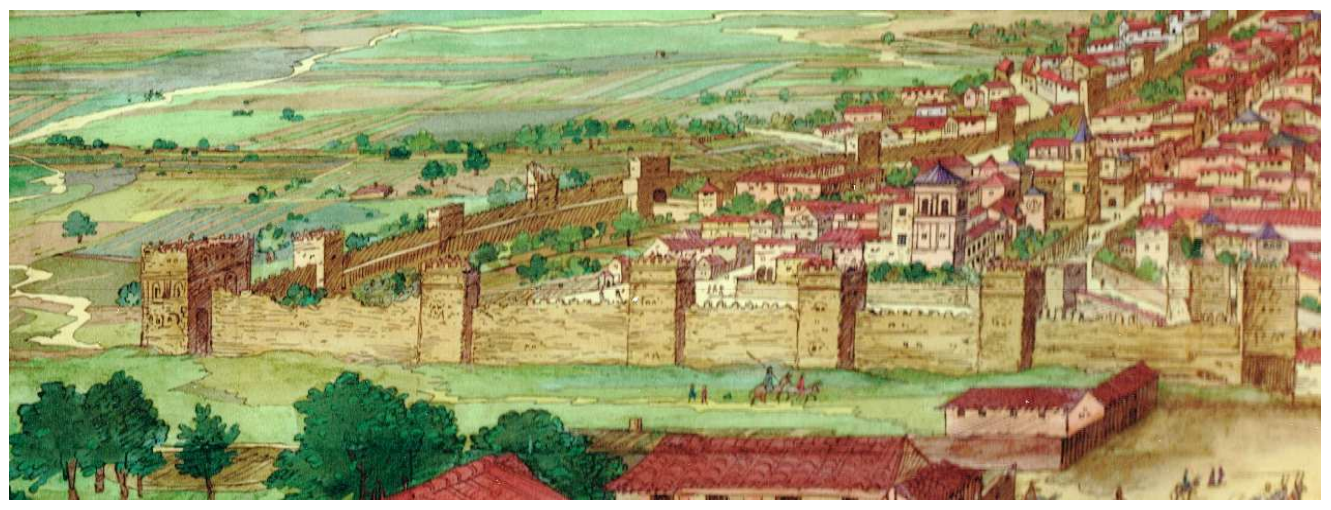

Fig. 1. Detalle de la vista de Alcalá de Henares realizada por Anton Van Der Wyngaerde realizada en 1565 (Österreichische Nationalbibliothek), donde aparece en primer término el recinto amurallado del alcázar arzobispal (Tratamiento y mejora de la imagen por José María de la Peña).

Las desamortizaciones de las propiedades de la iglesia del siglo XIX, acabarían haciendo que el conjunto residencial de los arzobispos de Toledo en Alcalá de Henares, se transformase en Archivo General de Reino en 1859. La mayor parte de la información histórica, sobre la residencia de los arzobispos de Toledo en Alcalá de Henares, junto con buena parte de la historia de este país, se pierden cuando el archivo ardió de forma fortuita, en el verano de 1939, una vez finalizada la Guerra Civil (San Luciano, 2009).

Esta circunstancia dificulta enormemente la investigación sobre los orígenes del alcázarpalacio y por supuesto sobre su sistema de abastecimiento e hizo que este cayese un total olvido.

\subsection{El sistema de abastecimiento del alcázar}

El alcázar disponía, no se sabe bien desde cuándo, de un sistema para el aprovisionamiento de agua (Eznarriaga, 1889). Sabemos ahora que se trata de la antiquísima técnica, denominada qanat, en su voz árabe.

Un qanat es un conjunto de galerías subterráneas, con unos pozos intercalados en un intervalo que varía habitualmente dependiendo de los casos, de 15 a $50 \mathrm{~m}$, que sirven para su construcción, nivelación y mantenimiento posterior. La sección de la galería tiene normalmente el tamaño justo que necesitaría una persona para su excavación de forma manual en mina, unos 0,6 
$\mathrm{m} x$ 1,7 m. La galería se construye interceptando un acuífero, del que se captaría el agua, que la propia galería, por gravedad, con una ligera pendiente, se encarga de transportar hasta su punto de consumo o hasta una red de distribución a base de acequias ó conductos. Por lo que la longitud de un qanat puede variar desde unos centenares de metros a decenas de kilómetros. La profundidad a la que se sitúan estas minas oscila en cada caso y varía desde unos pocos metros, a más de un centenar, en algún caso documentado.

Este sistema de abastecimiento propio fundamentalmente de las regiones áridas o semiáridas, tiene su origen en Persia, en lo que hoy es Irán, hace más de tres mil años (Hermosilla, 2006), donde siguen siendo hoy en día fundamentales en el abastecimiento de muchas poblaciones y también grandes ciudades como Teherán (Beckett, 1952). Los qanawat (en plural) están detrás, en la mayor parte de las ocasiones, de la cuasi milagrosa aparición de agua en los oasis del desierto, que tanto sorprendía a los exploradores franceses y británicos del siglo XIX (Martin, 1908). Ya en España existen casos singulares como el de Guadalajara (Diges, 1890) y Madrid. Esta última que se abastecía de los conocidos en esta zona, como "viajes de agua", desde su fundación en el siglo IX, hasta al menos el siglo XIX, momento en el que empezaron a ser sustituidos por las aguas del Canal de Isabel II, procedentes del río Lozoya (Solesio, 1975).

\section{La investigación del viaje de agua}

\subsection{Antecedentes}

Eran muy pocas las referencias que existían en la bibliografía referentes al sistema de abastecimiento del conocido como Palacio Arzobispal y completamente desconocido su trazado. Existe una mención al sistema de abastecimiento propio del alcázar prelaticio, que sitúa su construcción hacia 1300 (CH Tajo, 1954; Abastecimiento de agua potable a la ciudad de Alcalá de Henares. Dirección General de Obras Hidráulicas, Ministerio de Obras Públicas), aunque no consta la fuente de donde se obtiene dicha información. La siguiente mención sobre su origen la realiza un arquitecto del Ministerio de Fomento, Arturo Mélida, que lo sitúa en el siglo XVI, también sin hacer referencia a la fuente de la información (AGA, leg. 31/8203, 1890; Proyecto de reforma de la conducción de aguas al palacio arzobispal (hoy archivo) de Alcalá de Henares).

"Los príncipes de la iglesia que sucesivamente fueron ocupando la silla episcopal de Toledo enriquecieron considerablemente su residencia señorial de Alcalá de Henares de cuya importancia da buena muestra el recinto fortificado que cierra su perímetro.

No podía faltar en tan importante palacio rodeado de huertas y jardines el agua primera necesidad de la vida y a ella atendieron con largueza dotándole de un caudaloso viaje cuyas obras debieron llevarse a cabo en el siglo XVI. A este fin recogieron en la llamada tierra de las monjas, así como en la vega del Camarmilla las que por galerías llevaron a converger en el "pozo de Canto Blanco" conduciéndolas de este punto por medio de tuberías a la arqueta que se denomina del Chorrillo, situada en el paseo del mismo nombre."

No es hasta 1594, cuando las referencias al sistema de minas de agua del conjunto arzobispal aparecen en el Archivo Histórico Municipal de Alcalá de Henares (AHMAH, leg. 1504/3, 1920; Expediente de licencia de acometida de aguas y correspondenci). Para localizar sus restos y su trazado ha sido necesario recurrir, además de las referencias documentales, procedentes de diversos archivos, a la planimetría, cartografías y fotografías históricas (Fernández Tapia, 2015).

\subsection{El papel del agua en el recinto}

La investigación sobre el viaje de agua de "El Chorrillo" o de "Palacio", como se le conocía, nos ha permitido conocer mejor el importante papel que han jugado sus aguas, tanto en la historia del propio edificio, como en la ciudad.

El viaje de agua hacía que el conjunto del alcázar-palacio de los arzobispos de Toledo fuese un lugar lleno de jardines y huertas (Fig. 2), según un documento donde se hace una relación de la distribución de las aguas del viaje sabemos 


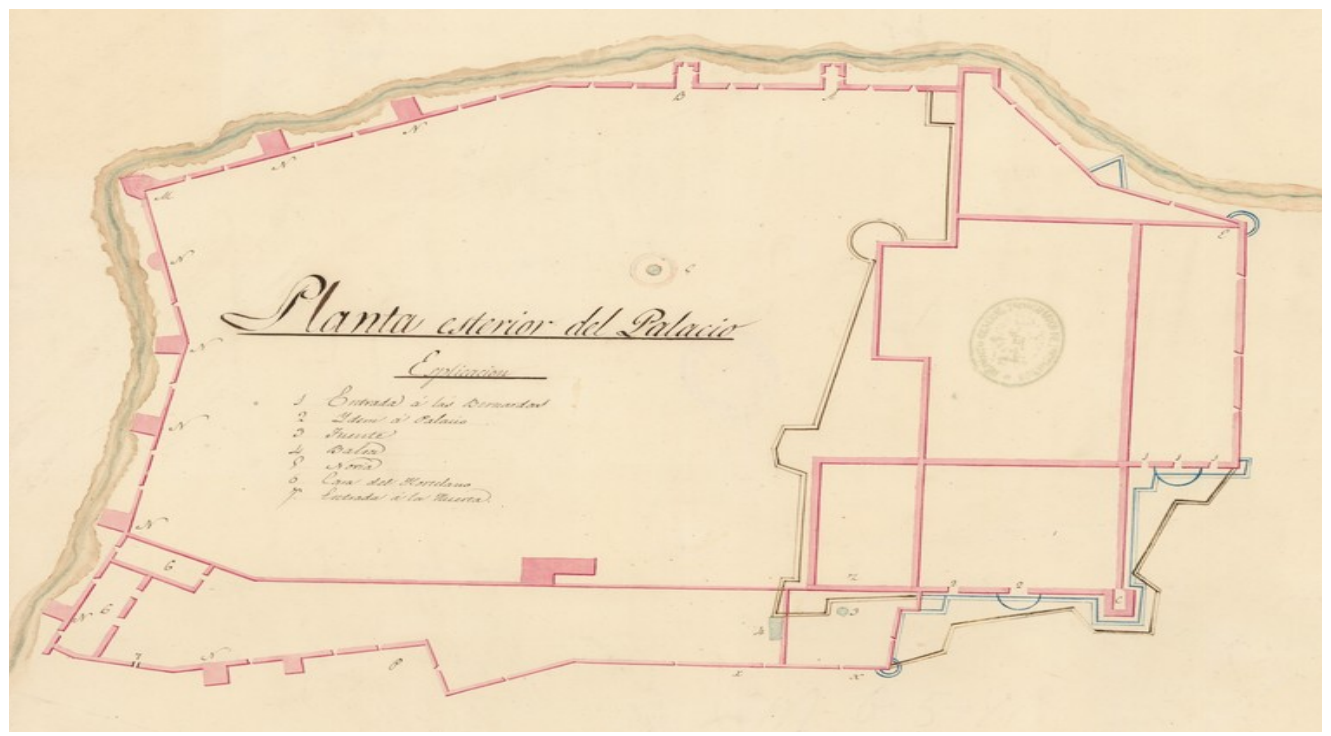

Fig. 2. Planta exterior del recinto amurallado del alcazar-palacio, donde se recogen elementos relacionados con el viaje de agua y la huerta a la que abastecía: fuente, balsa, y noria (Pedro Ortiz de Pinedo 1837, Cartoteca Militar).

qué puntos eran abastecidos y su caudal, dentro de sus murallas (Fig. 3), (AHMAH, leg. 1511/60, 1899; Correspondencia entre el ayuntamiento y el arzobispo de Toledo etc):

$\begin{array}{ll}\text { Jardín de la aleluya } & 2 \text { reales } \\ \text { Cocinas } & \text { (hoy nada) } \\ \text { Jardín del Vicario } & 2 \text { reales } \\ \text { Jardín de la Noguera } & 3 \text { reales } \\ \text { Estanque de la Alameda } & 3 \text { reales } \\ \text { Jardín Grande } & 3 \text { reales } \\ \text { Cenador del Jardin Grande } & 2 \text { reales }\end{array}$

\subsection{Su importancia en la ciudad}

Con el paso del tiempo, las cesiones de agua para fuentes pública, por parte del arzobispado, hicieron que el viaje de El Chorrillo, acabase convirtiéndose en una de las principales infraestructuras de abastecimiento de la ciudad (AHMAH, leg. 1511/60, 1899).

De igual forma, los arzobispos de Toledo fueron haciendo concesiones de porciones del caudal de sus aguas, más o menos graciosamente, a diversas órdenes religiosas, que se había ido implantando en la entonces villa, entre los siglos XVI al XIX, (AHMAH, leg. 1511/60, 1899):
Concesiones antiguas:

Religiosas Bernardas

5 reales

Religiosas S. Juan Peniten. $\quad m d$. cuartillo

Religiosas Magdalenas

Religiosas Sta. Clara

medio real

Religiosas de la Imagen

3 reales

Religiosos dominicos, cárcel 2 reales

Religiosos capuchinos,

un cuartillo

Religiosos San Felipe

medio real

\subsection{La importancia del viaje de agua en su transformación en Archivo Central de Reino}

Uno de los aspectos más importantes tenidos en cuenta para transformar el Palacio Arzobispal en Archivo General del Reino en 1859, era que contaba con un viaje de agua propio, que fue aprovechado para abastecer el sistema de bocas de incendios con que se dotó al edificio (AHN, Diversos Titulos Familias, 3773, Exp.17_015, 1880; Proyecto de distribución de aguas y bocas de incendio del Archivo Central del Reino. Real Academia de Bellas Artes de San Fernando), además de para la limpieza y para el riego de las huertas de las que los empleados del Archivo disfrutaban dentro del recinto (AHMAH, leg. 961/3, 1851; Expediente de licencia para conducción de agua a las fuentes públicas). 


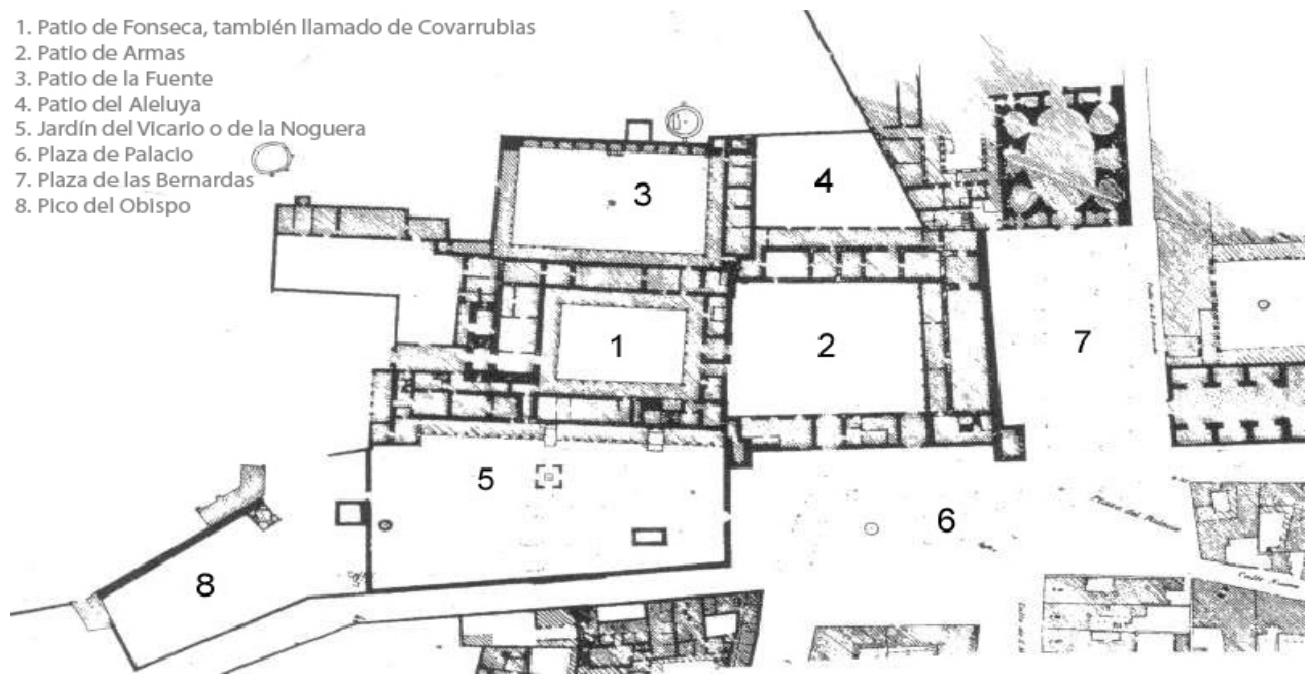

Fig. 3. Plano de planta de las diferentes zonas de conjunto del palacio arzobispal por donde discurría el viaje de agua, alimentando fuentes y depósitos (Abel Martínez y J. L. Vigara, 2009).

\section{Conclusiones}

Los viajes de agua son infraestructuras históricas ocultas. Tan sólo puntualmente, repartidos a lo largo de su trazado, las tapas algunos de sus pozos y arcas y sus puntos de servicio (fuentes, abrevaderos y lavaderos), son visibles, el resto discurre por el subsuelo. El sistema basado en la técnica árabe del qanat, que abastecía al alcázar de los arzobispos de Toledo en Alcalá de Henares, fue utilizado al menos, hasta mediados del siglo XX, cuando poco a poco fue sustituido por el nuevo abastecimiento que traía aguas del río Sorbe, cayendo en pocos años en el más absoluto olvido.

La vigente ley de Patrimonio Histórico de la Comunidad de Madrid, reconoce el papel que los viajes de agua en nuestra historia y los declara Bienes de Interés Patrimonial. También la UNESCO recomienda su protección y como ejemplo, los aflaj de Omán (2006) y los qanat de Irán (2016), han sido reconocidos con el título de Patrimonio Mundial.

La importancia del agua para la vida es proverbial y nuestros antepasados, a todos los niveles, debían realizar grandes esfuerzos para disponer de ella. El líquido elemento condicionaba enormemente, en mucha mayor medida que en la actualidad, su desarrollo vital. Se hacía pues necesario recuperar para la memoria colectiva, todo lo relativo a estos viajes de agua (origen, trazado, situación actual, etc.), y así poder relacionarlo con el alcázar y su historia.

Las investigaciones realizadas, que propician esta publicación, ha unido por un lado la investigación histórica, el estudio de la cartografía antigua, y la localización de sus restos sobre el terreno, utilizando entre otras, las más modernas técnicas de prospección, como el geo-radar o LIDAR. Toda la información encontrada se ha interrelacionado y georreferenciado, con respecto a la cartografía actual (Fig. 4), situando de esta manera, los viajes de agua, en relación no solo a la historia de la ciudad, sino también con respecto a su trazado urbano.

\section{Notas}

AGA, Archivo General de la Administración.

AHMAH, Archivo Histórico Municipal de Alcalá de Henares.

AHN, Archivo Histórico Nacional. 


\section{Agradecimientos}

Este artículo recoge resultados obtenidos en el Proyecto de Investigación: "Localización, inventario, catalogación y difusión de los viajes de agua históricos de Guadalajara para su puesta en valor, VAGUADA"; financiado por la Consejería de Educación, Cultura y Deportes de la Junta de Comunidades de Castilla La Mancha, de 18/12/2018 y cofinanciados por el Fondo Europeo de Desarrollo Regional (Feder). Extracto BDNS (Identif.): 430464. [2018/15051]. A su vez, también recoge resultados obtenidos en el proyecto de investigación titulado: "Estudio Multidisciplinar para la Puesta en Valor, Difusión y Propuesta de Protección de los Viajes de Agua Históricos del Campus Científico Tecnológico de la Universidad de Alcalá", VAHCUA; financiado por el Vicerrectorado de Investigación de la Universidad de Alcalá.

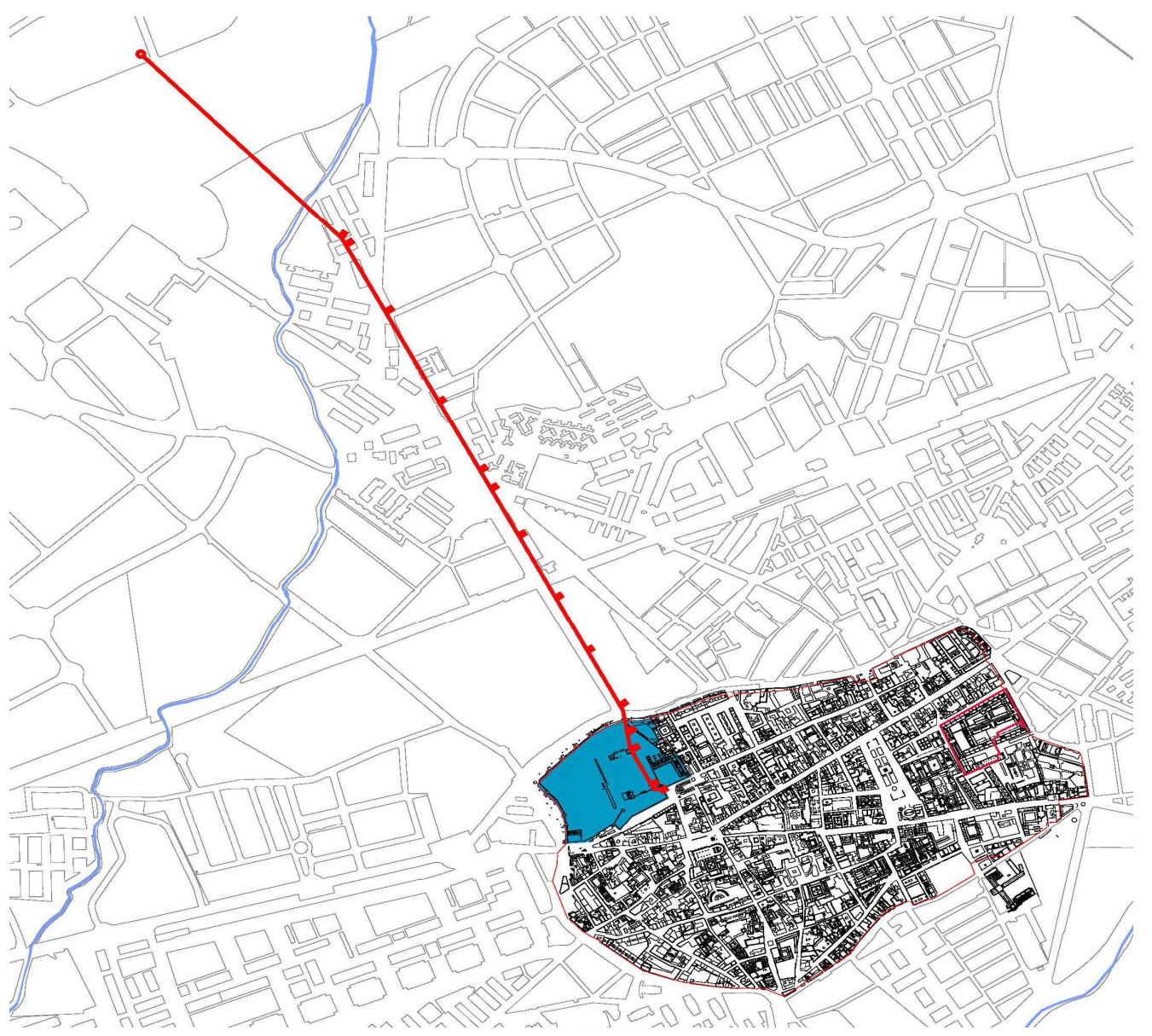

Fig. 4. Plano parcial del trazado urbano actual de Alcalá de Henares, donde se aprecia el recinto amurallado del alcázar de los arzobispos de Toledo (en azul), en la parte noroeste del casco histórico y el trazado del viaje de agua con sus arcas de registro y reparto (en rojo), según la investigación llevada a cabo (Autores, 2019). 


\section{Bibliography}

Beckett, P. (1952). "Qanats in Persia", Journal of the Iran Society, Londres, 1, pp. 125- 133.

Colin, S. (1932). La Noria marocaine et les machines hydrauliques dans le monde árabe, Larouse, p. 40.

Consuegra Gandullo, A. (2014). "Evolución Arquitectónica del Palacio en la Edad Media y el Renacimiento", in $E l$ Palacio Arzobispal de Alcalá de Henares. Historia y Arquitectura, pp. 49-92.

Diges Antón, J. (1890). Guía de Guadalajara, Guadalajara, p. 288.

Eznarriaga é Iglesias, A. (1889). Reseña medico topográfica del Alcalá de Henares, Madrid.

Fernández Tapia, E. (2015). Las galerías drenantes de Alcalá de Henares, Tésis Doctoral, Universidad de Alcalá, Alcalá de Henares.

Hermosilla Pla, J. (2006). Las galerías drenantes del sureste de la península Ibérica. Uso tradicional del agua y sostenibilidad en el Mediterráneo español, Ministerio de Medio Ambiente, Madrid.

Málaga Galíndez, J.M. (1988). Alcalá de Henares, arquitectura del siglo de oro, Institución de Estudios Complutenses, Alcalá de Henares.

Martin, A.G.P. (1908). A la frontière du Maroc. Les oasis sahariennes, Algérienne, Alger, t. 1, f. Vi, p. 410.

Pavón Maldonado, B. (1982). Alcalá de Henares Medieval: Arte Islámico y Mudejar, Consejo Superior de Investigaciones Científicas (CSIC), Instituto de Estudios Árabes Miguel Asín, Madrid.

San Luciano, J.M. (2009). El incendio y destrucción del Archivo General Central Alcalá de Henares, 1939, Domiduca Libreros, Madrid.

Sánchez Montes, A.L.; Rascón Marqués, S.; Bonor Villarejo, J.L. (2011). Guía-Catálogo, del Burgo de Santiuste y antiquarium y paseo arqueológico del Palacio Arzobispal. Una introducción a la Alcalá medieval y a su Palacio Arzobispal, Guías arqueológicas de Alcalá de Henares, 2, Ayuntamiento de Alcalá de Henares, Alcalá de Henares.

Sánchez Montes, A.L.; Rascón Marqués, S. (2013). "Complutum", in Escudero Escudero, F.A.; Gálvez Izquierdo, Mª drs., Las cloacas de Cesar Augusta, Institución Fernando el Católico, Zaragoza, pp. 370-386.

Solesio de la Presa, M.T. (1975). Antiguos Viajes de agua de Madrid, Monografías del Instituto Eduardo Torroja, Consejo Superior de Investigaciones Científicas, Madrid.

UNESCO. (2019). Word Heritage List, in https://whc.unesco.org/en/list/ (16/09/2019).

Vallejo Girvés, M, (1992). Fuentes históricas para el estudio del Complutum romano, Alcalá de Hres.

Vázquez Madruga, M.J. (1993). La donación de Alcalá a los arzobispos de Toledo. Institución del señorío de Alcalá. Documento fundacional, en Alcalá 1293: una villa universitaria de la Edad Media, p. 49-57. 